\title{
Production control and replenishment strategy with multiple suppliers
}

\author{
ADNENE HAJJI ${ }^{1}$, ALI GHARBI ${ }^{2}$, JEAN-PIERRE KENNE ${ }^{2}$, ROBERT PELLERIN ${ }^{3}$ \\ ${ }^{1}$ OSD-LAVAL \\ UNIVERSITE LAVAL, QUEBEC (Qc), Canada \\ adnene.hajji@,osd.ulaval.ca \\ ${ }^{2}$ LCCSP-LITP-ETS \\ ÉCOLE DE TECHNOLOGIE SUPERIEURE, Mtl (Qc), Canada \\ ali.gharbi@etsmtl.ca; Jean-pierre.kenne@,etsmtl.ca \\ ${ }^{3}$ MGI-POLY MONTREAL \\ ÉCOLE POLYTECHNIQUE DE MONREAL, Mtl (Qc), Canada \\ robert.pellerin@polymtl.ca
}

\begin{abstract}
This paper considers the joint supplier selection, replenishment and manufacturing control problem in a dynamic stochastic context. This problem is characterized by conflicting interests between suppliers, the manufacturer, and clients, which raise the need for coordination and information sharing. This paper contributes to the discourse mainly by developing and resolving an integrated mathematical model leading to information sharing strategies for supplier selection, replenishments and production activities. This is an optimal control problem with state constraints and hybrid dynamics. A dynamic stochastic model is thus proposed, and the optimality conditions obtained are then solved numerically. It is shown that the problem considered leads to a modified state-dependent multi-level (s, S) policy for the supplier selection and replenishment strategy and a base-stock policy for the production activities. The fact that these control policies are coupled confirms the necessity of considering the interactions present in the system in an integrated model. The obtained results show clearly that it is always profitable to consider multiple suppliers to make replenishment and production decisions. Moreover, it is shown that the availability rates of the supply chain actors and the replenishment lead time are important parameters to consider when choosing the best supplier.
\end{abstract}

Keywords - Stochastic optimal control, production rates, replenishment policy, supplier selection strategy.

\section{INTRODUCTION}

In today's ever-changing markets, most manufacturing enterprises operate under highly competitive pressures, and this reality has promoted the establishment of network organisations. Supply chain management (SCM) strategies are used to administer such networks. To accomplish the shortand long-term objectives of SCM (e.g., productivity improvement, inventory reduction; customer satisfaction, market share, and profits improvement), tight coordination is needed among organizations in supply chains (Tan et al. (1998), Lee et al. (2001)). For manufacturers, the supply (or purchasing) function is widely recognized as constituting a very important key for improving performances in the supply chain Chuang (2004). In fact, since suppliers are in effect external organizations for manufacturers, coordination with suppliers is not an easy matter unless cooperation and information exchange systems are integrated (Lee et al. (2001), Chan and Chan (2009)). In the research literature, these issues are considered from two perspectives: the first one aims to develop new methodologies leading to improved supplier selection processes, while the second seeks new and improved replenishment strategies in the presence of multiple suppliers.

Several studies have investigated the supplier selection process with a view to improving the supply chain performance over the long term. We refer the reader to Choi and Hartley (1996), Verma and Pullman (1998) and Ghodsypour and O'Brien (1998) and their references for a complete literature review of this issue. For a considered class of product, assume that a set of potential suppliers have been selected. To deal with the suppliers-manufacturer-clients relationships in a mid-term or short-term perspective, several issues should be addressed including: from the pre-selected set of suppliers, who can respond efficiently to a current order? What is the order quantity to place? At what time should the order be placed? What is the best production strategy to apply to respond to the clients? These issues are difficult to resolve due to the conflicting objectives and the presence of random phenomena.

In the literature, these issues have been addressed only partially. Moreover, several approaches were developed by formulating the problem with a given replenishment policy. In Chuang (2004), the suppliers-manufacturer relationship has been studied and the order allocation problem solved through a goal programming approach. Essentially, the problem consists in determining the economic order quantities that should be placed with several suppliers in order to tackle multiple objectives. Similarly Syam and Shetty (1998) considered a situation in which a purchasing manager must determine the timing and size of replenishment orders for a number of items, each of which may be supplied by several vendors, each with 
capacity constraints. The model considers multiple suppliers with limited capacity, transportation economies, and quantity discounts. An integrated branch and bound procedure is proposed to solve the model. In a dynamic stochastic context Parlar and Perry (1995) considered an inventory model in which the supplier's availability process is presented as a twostate continuous time Markov chain. The problem was to determine the reorder point and the order quantity when the system is in the ON and OFF states. Similarly, Parlar and Perry (1996) analyzed the same features with single and multiple suppliers. In that paper (Parlar and Perry (1996)), the authors stated that « ideally one should use dynamic programming to discover the optimal policy that may differ from the one used in this article ».

To deal with this issue several researches have attempted to find the optimal control strategy in a dynamic stochastic context. In the class of a single stage supply chain facing deterministic lead time and random demands, Zhang (1996) analyzed a model with three suppliers and lead times that differ by one and two periods. The optimal policy is explicitly stated. In addition, simple heuristic ordering policies are discussed and a heuristic framework, based on newsvendor considerations is developed in order to provide decision support for finding appropriate replenishment policy parameters. Within the class of continuous review policies, Moinzadeh and Nahmias (1988) analyzed an extension of the (s, Q) policy. Within such a policy, an economic lot Q of raw materials is ordered when the downstream inventory level reaches s. In their model, the objective is to minimize long-run average costs. The suggested ordering policy is an (s1, s2, Q1, Q2) policy based on on-hand stock, and consists in placing regular order Q1 when s1 is reached. If, within the replenishment lead time of the regular order, the emergency reorder point s2 is reached, an order of size Q2 is placed. Similarly, Johansen and Thorstenson (1998) analyzed a similar model where regular replenishments with a long lead time are controlled by a continuous review (s, Q) policy.

Recently, Hajji et al. (2009) addressed the joint replenishment and production control problem in a two stage unreliable supply chain (i.e., one unreliable supplier, one unreliable transformation stage). The problem is tackled with a dynamic stochastic formulation and the robustness of the developed policies including the production and supply activities was demonstrated.

In order to better approach the real context of supply chains, two elements are considered in the present paper to extend the findings of Hajii et al. (2009): the multiple supplier case and the replenishment lead time. In this context, it should be noted that in the literature the replenishment lead time was considered only in the single stage supply chain were research studies have almost exclusively used continuous review (s, Q) policies and focused on the determination of the optimal number of suppliers, the reorder point, the total order quantity and its allocation among the suppliers. These studies have focused on the statistical aspects with a view to examining the advantage of placing orders with multiple suppliers. In Fong et al. (2000) this issue is considered for effective lead times and stock-out probabilities for a dual sourcing inventory system facing normally distributed demands and lead times distributed according to mixtures of Erlang distributions. For an extended review of inventory models with multiple supply options we refer the reader to Minner (2003).
In this paper, an integrated production, supplier selection and replenishment control problem of a stochastic supply chain is considered. To investigate the interaction aspect of this class of supply chains in a stochastic context, information sharing control policies are required. While availability, capacity and delivery performances are implicitly considered, an important performance criterion, namely, the expected discounted cost over an infinite horizon, is explicitly considered. This criterion includes ordering, inventories/backlog and transformation costs. A stochastic dynamic programming problem is thus formulated. The formulation developed includes the raw material inventory constraint aspect as well as the hybrid nature of the problem. The structure of the solution, under appropriate conditions, is obtained by using the fact that the value function is the unique viscosity solution to the associated Hamilton Jacobi Bellman (HJB) equations. Because an analytical solution of HJB equations is not generally available, a numerical approach is adopted to illustrate the structure of the control policy. Numerical examples and sensitivity analysis are then conducted to achieve a close approximation of the optimal control policy. Moreover, a comparative study between the multiple and single supplier cases is detailed.

The remainder of the paper is organized as follows. Section 2 presents a formulation of the optimal production and supply problem. Section 3 provides the results obtained aimed at illustrating the optimal control policy structure. A complete characterization of the production and supply policy is reported in section 4 . The comparative study is outlined in section 5 . The paper is concluded in section 6 .

\section{NOTATION AND PROBLEM FORMULATION}

\subsection{Notation}

The following notation is used.

$x$ : Raw material inventory level

$y$ : $\quad$ Finished product inventory / backlog level.

$d$ : $\quad$ Finished product demand rate

$p$ : Manufacturing production rate.

$p^{\max }$ : Maximum manufacturing production rate.

$x_{\text {cap }}$ : Raw material stock capacity.

$\theta_{i}: \quad$ Raw material $i^{\text {th }}$ order reception instants.

$\delta^{j}$ : Delay between order decision and its reception.

$Q_{i}^{j}():. i^{\text {th }}$ Order quantity corresponding to instant $\theta_{i}$, ordered from supplier $j$.

$q_{\alpha \beta}$ : Transition rates from modes $\alpha$ to $\beta$.

$K^{j}$ : Ordering cost of supplier $j$.

$c_{R}^{j}$ : Unit raw material cost from supplier $j$.

$c_{R}^{H}$ : Unit raw material holding cost.

$c_{F}^{H}$ : Unit finished product holding cost.

$c_{F}^{B}$ : Unit finished product backlog cost.

$c_{R F}^{T}$ : Unit of raw material transformation cost.

\subsection{Problem formulation}

The supply chain under study (illustrated in Figure 1) consists of an unreliable manufacturing system supplied by multiple unreliable suppliers. The whole system faces a one family product demand. 
In this supply chain the manufacturer (Stage 2) orders raw materials from the more competitive supplier (Stage 1). The main criteria for supplier choice are price and supplier service (lead time and reliability). In this study, we assume that the supplier service will lead to indirect costs such as costs for holding a safety inventory to meet supply and production variability. Then, through the production processes, the manufacturer converts the raw materials to finished goods which are delivered to the clients (Stage 3). Hence, between the first and the second stages, we have raw material holding cost, and raw material and ordering costs. Between the second and the third stages, there are production, holding and backlog costs.

The evolution of the supply chain under study changes with time (i.e., evolves under conditions of uncertainty). To formulate the optimization problem in a dynamic stochastic context, one needs to characterize the state of the system at each instant $t$. In our case, we have defined the state of the system at time $t$ using three components. A continuous part which describes the cumulative surplus level (inventory if positive and backlog if negative) and measured by $y(t)$. A piecewise continuous part which describes the raw material level and measured by $x(t)$. This part faces continuous downstream demand (i.e., manufacturing production rate) and an impulsive upstream supply when a $Q_{i}^{j}$ lot of raw material is received from supplier $j$ at instant $\theta_{i}+\delta^{j}$. Even if the transformation manufacturing system is available, it cannot proceed with parts when $x(t)$ is equal to zero. Let $0 \leq x(\mathrm{t}) \leq x_{\text {cap }}$ be the capacity constraint of the raw material stock. A discrete part which describes the whole system state (supplier and transformation manufacturing system) and given as follows.

The operational mode of the suppliers and the transformation manufacturing system at time $t$ can be described by the random variables $\xi_{k}(t), k=1, \ldots, n$ and $\xi_{n+1}(t)$, with value in $M_{k}=\{1,2\}, k=1, \ldots, n+1$, respectively, where

$$
\begin{aligned}
& \zeta_{k}(t)=\left\{\begin{array}{ll}
1 & \text { the supplierk is available } \\
2 & \text { the supplier kis unavailable. }
\end{array}, k=1, \ldots, n\right. \\
& \zeta_{n+1}(t)= \begin{cases}1 & \text { the transformation stage is available. } \\
2 & \text { the transformation stage is unavailable. }\end{cases}
\end{aligned}
$$

The transition rates matrix of the stochastic processes $\xi_{k}(t), k=1, \ldots, n$ and $\xi_{n+1}(t)$ are denoted by
$\mathrm{T}_{i}=\left\{q^{i} \alpha \beta\right\} i=1, \ldots, n+1, \quad$ with $\quad q^{i} \alpha \beta \geq 0$ if $\quad \alpha \neq \beta \quad$ and $q^{i} \alpha \alpha=-\sum_{\beta \neq \alpha} q^{i} \alpha \beta$, where $\alpha, \beta \in M_{i}$. The transitions rates matrix $\mathrm{T}_{i}$ is expressed as follows:

$$
\mathrm{T}_{i}=\left|\begin{array}{cc}
-q_{12}^{i} & q^{i}{ }_{12} \\
q^{i} 21 & -q^{i} 21
\end{array}\right|
$$

Without loss of generality, for a transformation stage facing two suppliers, the operational mode of the whole system can be described by the random vector

$$
\begin{aligned}
& \xi(t)=\left(\xi_{1}(t), \xi_{2}(t), \xi_{3}(t)\right) \text { taking values in } \\
& M=M_{1} \times M_{2} \times M_{3} .
\end{aligned}
$$

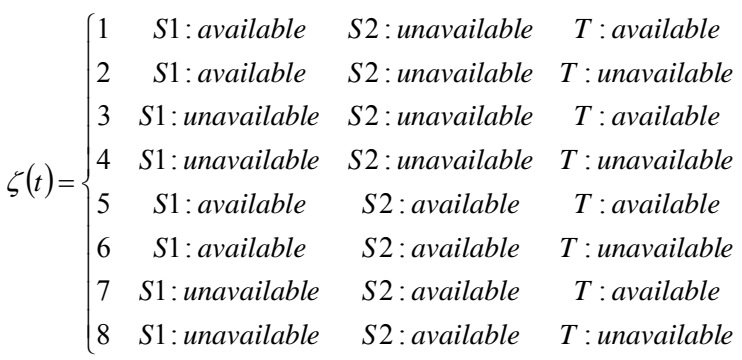

Such that S1, S2 and T denote supplier 1, supplier 2 and the transformation stage respectively.

For the supply chain considered, the state space is given by $(x, y, \alpha)$ such that:

$x \in\left\lfloor 0, x_{\text {cap }}\right\rfloor ; y \in R ; \alpha \in M, \quad$ let $\quad S=\left\lfloor 0, x_{\text {cap }}\right\rfloor \times R \quad$ and $\partial S=\left\{0, x_{\text {cap }}\right\} \times R$ and $\left.S^{0}=\right] 0, x_{\text {cap }}[\times R$ the interior of $S$.

The dynamic of the stock levels $x(\mathrm{t})$ and $y(\mathrm{t})$ is given by the following differential equations.

$\dot{y}(t)=p(t, \alpha)-d, y(0)=y, \forall t \geq 0$

$\dot{x}(t)=-p(t, \alpha), x(0)=x, \forall t \in] \theta_{i}, \theta_{i+1}[$

$x\left(\left(\theta_{i}+\delta^{j}\right)^{+}\right)=x\left(\left(\theta_{i}+\delta^{j}\right)^{-}\right)+Q_{i}^{j}(\alpha), i=1, \ldots, N . j=1, \ldots, n$

Where $x, y$ denote the surplus levels at the initial time, and $\left(\theta_{i}+\delta^{j}\right)^{-}$and $\left(\theta_{i}+\delta^{j}\right)^{+}$denote the negative and positive boundaries of the $i^{\text {th }}$ receipt instant from supplier $j . \mathrm{N}$ is the number of the received orders in the whole horizon.

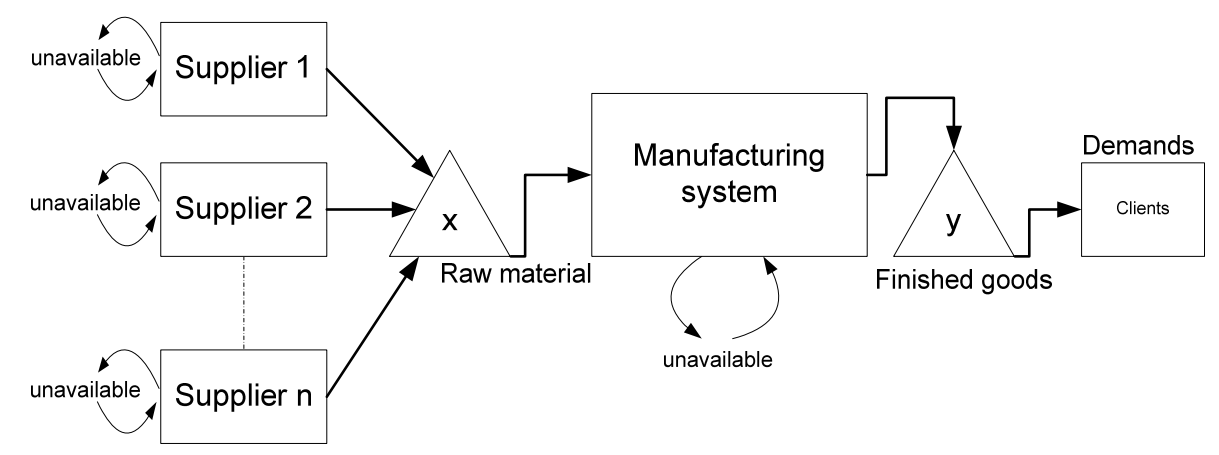

Figure 1. Unreliable three stage supply chain 
At any given time, the manufacturing production rates and the order quantities must satisfy the production and supply capacity constraints.

$$
\begin{aligned}
& 0 \leq p(t, \alpha) \leq p^{\max } \\
& 0 \leq x\left(\theta_{i}+\delta^{j}\right)+Q_{i}^{j}(t) \leq x_{c a p}, i=1, \ldots, N, j=1, \ldots, n
\end{aligned}
$$

Our decision variables are the manufacturing production rate $p(\cdot)$ and a sequence of supplier selection and supply orders denoted by $\Omega=\left\{\left(\theta_{i}, \lambda(i), Q_{i}^{\lambda(i)}\right), \ldots,, i=1, \ldots, N, \lambda()=1,. \ldots, n\right.$. With $\left(\theta_{i}, \lambda(i), Q_{i}^{\lambda(i)}\right)$ defined by the time $\theta_{i}$ at which the order is placed, the selected supplier $\lambda(i)$ and the order quantity $Q_{i}^{\lambda(i)}$. Let $A(\alpha)$ denote the set of admissible decisions $(\Omega, p()$.$) such$ that:

$$
A(\alpha)=\left\{\begin{array}{l}
(\Omega, p(t)), 0 \leq p(t, \alpha) \leq p^{\max }, \\
0 \leq x\left(\theta_{i}+\delta^{j}\right)+Q_{i}^{j}(t) \leq x_{\text {cap }}, i=1, \ldots, N, j=1, \ldots, n
\end{array}\right\}
$$

To penalize the raw material holding cost, the production rate and the finished product inventory (backlog) cost, we define the cost rate function $g($.$) as follows:$

$$
\begin{aligned}
& g(x(t), y(t), p(t, \alpha))=c_{R}^{H} \cdot x+c_{F}^{H} \cdot y^{+}+c_{F}^{B} \cdot y^{-}+c_{R F}^{T} \cdot p(.), \\
& t \in] \theta_{i}, \theta_{i+1}[
\end{aligned}
$$

Where, $y^{+}=\max (0, \mathrm{y}(\mathrm{t}))$ and $y^{-}=\max (-\mathrm{y}(\mathrm{t}), 0)$.

$z=(x(t), y(t)) \in S$.

In addition, we define the cost function which penalizes the supply order from the selected supplier $\lambda$ at time $\theta_{i}$. This function is defined as follows:

$$
\begin{aligned}
& R\left(Q_{i}^{\lambda}, \lambda, \alpha\right)=K^{\lambda} \operatorname{Ind}\left\{t=\theta_{i}\right\}+c_{R}^{\lambda} \cdot Q_{i}^{\lambda}+ \\
& E_{\alpha} \int_{0}^{\delta^{\lambda}} e^{-\rho t} g(x-p(t, \alpha) t, y+p(t, \alpha)-d t, p(t, \alpha)) d t
\end{aligned}
$$

The total cost $J($.$) can be defined by the following expression:$

$$
J\left(x, y, p, \theta_{k}, \lambda, Q_{k}^{\lambda(k)}, \alpha\right)=E\left[\begin{array}{l}
\int_{0}^{\infty} e^{-\rho t} g(x, y, p) d t+ \\
\sum_{k=0}^{\infty} e^{-\rho \theta_{k}}\left(K^{\lambda(k)}+c_{R}^{\lambda(k)} \times Q_{k}^{\lambda(k)}\right)
\end{array}\right]
$$

Where $E$ is the conditional expectation given the condition $(x, y, \alpha)$.
The control problem considered here is to find $\left(\Omega^{*}, p^{*}\right) \in A(\alpha)$ which minimizes $J($.$) given by (5) subject to$ (1) - (3). This is a feedback control that determines the production rate and the supply decisions (i.e., supplier selection and supply order) as a function of the system state. The value function of such a stochastic optimal control problem is given by:

$$
v(x, y, \alpha)=\inf _{(\Omega, p) \in A(\alpha)} J\left(x, y, p, \theta_{k}, \lambda, Q_{k}^{\lambda(k)}, \alpha\right)
$$

\subsection{Optimality conditions}

Based on the dynamic programming principle, the resulting optimality conditions are formally given by equation (7):

Where $(v)_{x}($.$) , denotes the gradients of v($.$) with respect to x$.

Because we are faced with a state-constrained problem, we need to shape the value function on the boundary of $S$. To state these boundary conditions, we follow the same theory introduced in [Capuzzo-Dolcetta and Lions, 1990], where it is shown that, for state constrained problems, we must consider the solution of the HJB equations as viscosity solution inside $S$ and viscosity super-solution on the boundaries (i.e., $\partial S$ ). The fact that $v(x, y, \alpha)$ is a viscosity super-solution on $\partial S$, plays the role of a boundary condition.

\section{NUMERICAL RESULTS}

To illustrate the supplier selection, replenishment and production policies several elements should be considered.

A. Parameters defining the manufacturing system: namely the maximum production rate $p^{\max }$, the demand rate $d$, the availability stochastic process $\xi_{3}(t)$, the raw material and finished product holding and backlog costs (i.e., $c_{R}^{H}, c_{F}^{H}, c_{F}^{B}$ ,$\left.c_{R F}^{T}\right)$.

B. Parameters defining the suppliers: namely the ordering costs $K^{1}, K^{2}$, raw material costs $c_{R}^{1}, c_{R}^{2}$, supply delay $\delta^{1}$, $\delta^{2}$ and availability stochastic processes $\xi_{1}(t), \xi_{2}(t)$.

C. Economic parameter: the discounted rate of the incurred cost (i.e., $\rho$ ).

Two steps are required in order to characterize the policies structure. The first consists in solving in an optimized manner (i.e., computation time and good choice of computation domain), the optimality conditions for a fixed set of parameters (i.e., A, B and C). The second step consists in carrying out sensitivity analysis in order to ascertain the validity of the results. These two steps are presented in the following sections.

$$
\min \left\{\begin{array}{c}
\min _{p \in A}\left\{(-p) v_{x}+(p-d) v_{y}+g(x, y, p)+\sum_{\beta \neq \alpha} q_{\alpha \beta}(v(x, y, \beta)-v(x, y, \alpha))\right\}-\rho v(x, y, \alpha) ; \\
\min _{\left(\lambda, Q^{\lambda}\right)} E\left\{R\left(Q^{\lambda}, \lambda, \alpha\right)+e^{-\rho \cdot \delta^{\lambda}} v\left(x+Q^{\lambda}-\delta^{\lambda} \cdot p, y+(p-d) \cdot \delta^{\lambda}, \alpha\right)\right\}-v(x, y, \alpha)
\end{array}\right\}=0
$$




\subsection{Data parameters}

To illustrate the optimal production and supply policies, experimentations were conducted with five cases of suppliers and manufacturing system availabilities (i.e., Sets I, II, III, IV and V). When the selected supplier is unavailable, the transformation stage must wait for a random length of time (random delay), which on average, is equal to the mean time for the supplier to become available, in addition to the fixed delay $\delta^{j}$. These cases studied (i.e., suppliers availabilities) showed the reaction of the transformation stage when faced with such a situation. Table 1 presents the data parameters.

Table 1. Data parameters

\begin{tabular}{|c|c|c|c|c|c|c|}
\hline & $p^{\max }$ & $d$ & $c_{R}^{H}$ & $c_{F}^{H}$ & $c_{F}^{B}$ & $c_{R F}^{T}$ \\
\hline Values & 2.5 & 2 & 0.3 & 0.35 & 5 & 0.1 \\
\hline & $K^{1}$ & $K^{2}$ & $c_{R}^{1}$ & $c_{R}^{2}$ & $\delta^{1}$ & $\delta^{2}$ \\
\hline Values & 3.5 & 1.5 & 1.5 & 0.5 & 0.1 & 0.4 \\
\hline
\end{tabular}

The transition rates matrices defining the supply chain stochastic processes for Sets I, II, III, IV and V are defined in Table 2 .

To ensure a clear characterization of the control policy, several elements were taken into consideration as part of the implementation process. Indeed, the selection, replenishment and production policies are each observed separately. For each policy, the relevant significant stock thresholds are first analyzed independently, then in connection with the others. For each numerical result, $p(x, y, \alpha)$ are the production policies of the transformation stage in the system state $\alpha$ and $A 1(x, y, \alpha)$ and $A 2(x, y, \alpha)$ are the replenishment policies in system state $\alpha$ from supplier 1 and 2 respectively.

\subsection{Results interpretation}

It follows from our numerical results that the replenishment policies are governed by a State- Dependent Economic Order
Quantity policy, SD-EOQ for short. This policy is governed by an order point and an economic order quantity, and these parameters depend on the complete state of the system $(x, y$ and $\boldsymbol{\alpha}$ ). The order point reflects the need to have a security raw material stock level to meet a possible random delivery delay when the supplier is unavailable or a big amount of backlog accumulates after a period of unavailability of the transformation stage. Moreover, it follows from the observed replenishment policies that the selection policy is governed by a State-Dependant Up-To-Levels policy, SD-UTL for short.

It should be noted that the preliminary analysis conducted under the fixed costs case (Table 1) and the five sets of suppliers and transformation availabilities (Table 2) enabled us to detect the impact of such stochastic parameters on the policies. As shown in Table 2, with a fixed availability of supplier 1 , the analysis conducted is aimed at determining the impact of a combination of higher availability of supplier 2 and lower availability of the transformation stage. A case study with low availabilities for the two suppliers (i.e., set V) is also conducted.

The results of the five sets show that the replenishment policies are governed by three sate-dependent factors for each supplier. These factors define the economic order quantities and the order points. The results of set I show that when the raw material level reaches certain zones of the inventory space, we have to order an economic quantity from supplier 1 or supplier 2, respectively.

Let $\left(S_{F 1}^{x, \alpha}, S_{F 1}^{y, \alpha}, Q_{F 1}^{\alpha}\right)$ the order point with respect to $x$, the order point with respect to $y$ and the order quantity if supplier 1 is selected, and $\left(S_{F 2}^{x, \alpha}, S_{F 2}^{y, \alpha}, Q_{F 2}^{\alpha}\right)$ the equivalent parameters if supplier 2 is selected. Figure 2 and 3 illustrate these factors.

Table 2. Transitions rates and availabilities

\begin{tabular}{||c|c|c|c|c|c|c||}
\hline SETS & $T_{1}$ & AVAIL. & $T_{2}$ & AVAIL. & $T_{3}$ & AVAIL. \\
\hline I & $\begin{array}{c}q_{12}^{1}=0.01 \\
q_{21}^{1}=0.33\end{array}$ & $97 \%$ & $\begin{array}{l}q_{12}^{2}=0.2 \\
q_{21}^{2}=0.1\end{array}$ & $33 \%$ & $\begin{array}{l}q_{12}^{3}=0.01 \\
q_{21}^{3}=0.33\end{array}$ & $97 \%$ \\
\hline II & $\begin{array}{c}q_{12}^{1}=0.01 \\
q_{21}^{1}=0.33\end{array}$ & $97 \%$ & $\begin{array}{l}q_{12}^{2}=0.02 \\
q_{21}^{2}=0.1\end{array}$ & $83 \%$ & $\begin{array}{l}q_{12}^{3}=0.01 \\
q_{21}^{3}=0.33\end{array}$ & $97 \%$ \\
\hline III & $q_{12}^{1}=0.01$ & $97 \%$ & $\begin{array}{l}q_{12}^{2}=0.2 \\
q_{21}^{2}=0.1\end{array}$ & $33 \%$ & $\begin{array}{l}q_{12}^{3}=0.02 \\
q_{21}^{3}=0.1\end{array}$ & $83 \%$ \\
\hline IV & $\begin{array}{c}q_{12}^{1}=0.01 \\
q_{21}^{1}=0.33\end{array}$ & $97 \%$ & $\begin{array}{l}q_{12}^{2}=0.02 \\
q_{21}^{2}=0.1\end{array}$ & $83 \%$ & $\begin{array}{l}q_{12}^{3}=0.02 \\
q_{21}^{3}=0.1\end{array}$ & $83 \%$ \\
\hline V & $q_{12}^{1}=0.3$ & $52 \%$ & $q_{12}^{2}=0.2$ & $33 \%$ & $\begin{array}{l}q_{12}^{3}=0.02 \\
q_{21}^{3}=0.1\end{array}$ & $83 \%$ \\
\hline \hline
\end{tabular}




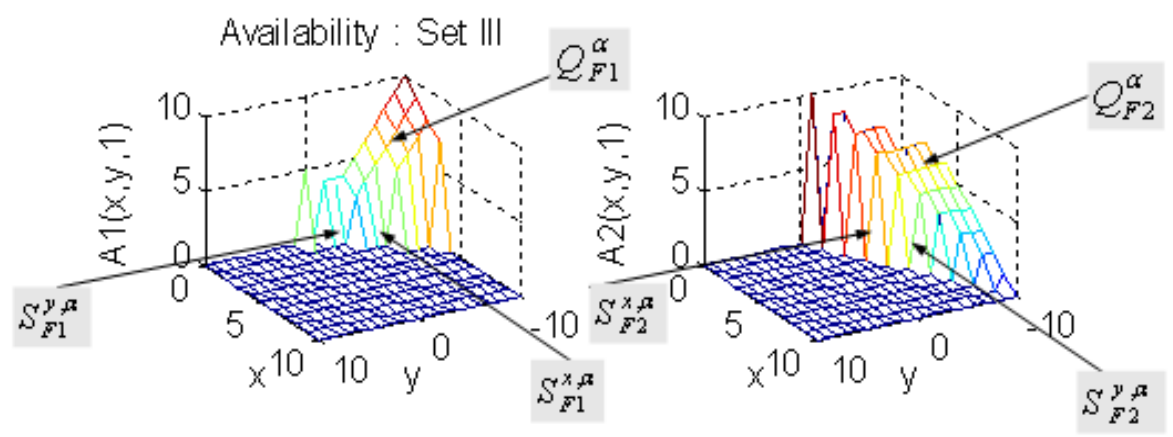

Figure 2. Replenishment policy boundaries
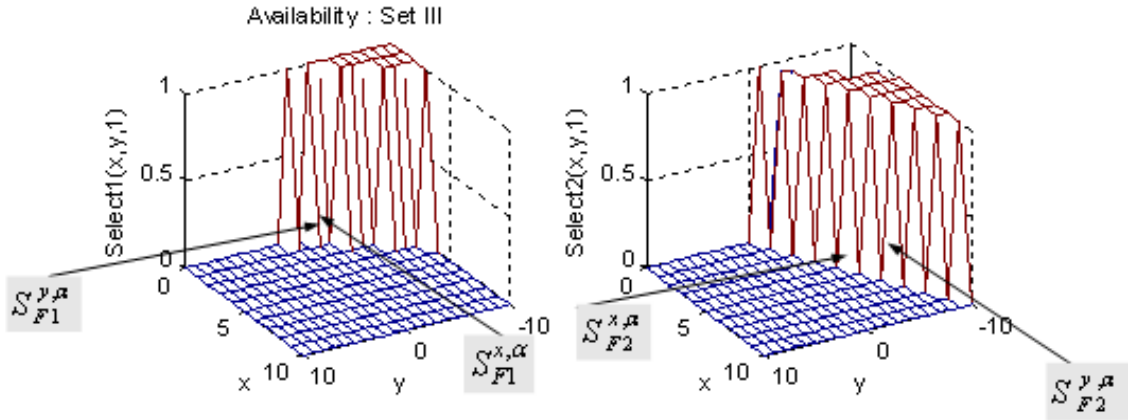

Figure 3 a: Select supplier 1 Figure 3 b: Select supplier 2 Figure 3. Selection policy boundaries

It is interesting to note that for the considered case, supplier 1, who has a better availability rate but a higher cost, is selected only in the system state 1 (i.e., supplier 1 available and supplier 2 unavailable). In this case, we choose to order from supplier 1 only if the finished product level $y$ is below a certain level of shortage. Otherwise, it seems more appropriate to order from the supplier with the lowest cost (i.e., supplier 2) for all other system states. Moreover, when the two suppliers are available, we observe a lower order point with respect to $x$. This means that the transformation stage does not have to forecast a large security raw material stock level.

If we observe the results of Set II (i.e., higher availability rate of supplier 2) in comparison with those of Set I, the results show that the zone reserved for replenishment from supplier 1 is smaller. This tendency shows that if the availability ratios of the suppliers become closer, the choice will be that which offers the lowest cost. The same observations apply for Set III in comparison with Set IV. On the other hand, if we observe the results of Set III (i.e., lower availability rate of the transformation stage) in comparison with those of Set I, the

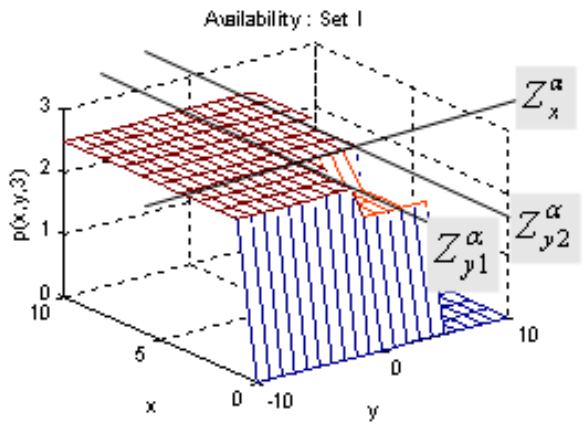

Figure 4. Production policy boundaries results show that we must order more while maintaining higher security levels (i.e., $S_{F 1}^{x, \alpha}, S_{F 1}^{y, \alpha}$ and $S_{F 2}^{x, \alpha}, S_{F 2}^{y, \alpha}$ ).

Furthermore, the results show that the resulting production policy divides the surplus space into three mutually exclusive regions: in zone 1, produce at the maximal rate; in zone 2, set the production rate equal to the demand rate, and in zone 3 , set the production rate to zero. Moreover, the results show that the area covered by these regions changes depending on the state of the whole system. These results point to a Modified statedependent multi level Base Stock Policy (MBSP for short) type of production control, given that at any time, the production rate is either at the minimum, demand or maximum level. With some approximations (i.e., we consider the average thresholds), to hedge against the existence of irregularities in the regions boundaries, we can define three state-dependent hedging levels which characterize the optimal production policy observed. Let $Z_{x}^{\alpha}, Z_{y 1}^{\alpha}$ and $Z_{y 2}^{\alpha}$ define these factors. Figure 4 illustrates how these factors delimit the stock space.

The results show that the production policy is governed by three hedging levels which delimit the areas where the production rate must be set to the maximum or to the demand rate. The values of these hedging levels depend on the state of the system. In the class of the hedging point policy, where the system must keep a finished product security level to hedge against periods of manufacturing system unavailability, our results are coherent. Moreover, we observe that the production at the demand rate appears in two states (i.e., State 3, and State 7). We can begin our analysis by considering the holding costs, which are different in the case considered, making it more profitable to keep the raw material in the upstream area when supplier 1 (i.e., which is the best from the availability point of view) is unavailable. However, when supplier 1 is available, the transformation stage must keep the stock in the 
downstream area in order to prevent the possibility of its next unavailability period. In addition, the results of Sets III and IV, with the same data parameters but with a lower availability rate of the transformation stage, confirm these observations. Indeed, the hedging levels are higher and the production rate is often at a maximum level, as compared to those of Set I, allowing the transformation stage to stock more products to hedge against its lower availability rate. In the same direction, the results of set V (i.e., low availabilities for supplier 1 and 2) show that the transformation stage must hedge against periods of suppliers unavailability by keeping higher raw material (i.e., $S_{F 1}^{x, \alpha}, S_{F 1}^{y, \alpha}$ and $S_{F 2}^{x, \alpha}, S_{F 2}^{y, \alpha}$ ) and finished product (i.e., $Z_{x}^{\alpha}$, $Z_{y 1}^{\alpha}$ and $Z_{y 2}^{\alpha}$ ) security levels as compared with those of set I to IV.

Finally, our numerical results show that the optimal policy is a combination of the MBSP, SD-UTL and SD-EOQ policies. The combined policy is completely defined by nine parameters, $Z_{x}^{\alpha}, Z_{y 1}^{\alpha}$ and $Z_{y 2}^{\alpha}$ for the production policy, $S_{F 1}^{x, \alpha}$, $S_{F 1}^{y, \alpha}$ and $Q_{F 1}^{\alpha}$ for the replenishment policy from supplier 1 and $S_{F 2}^{X, \alpha}, S_{F 2}^{y, \alpha}$ and $Q_{F 2}^{\alpha}$ for the replenishment policy from supplier 2. To ascertain the validity of those results, a sensitivity analysis has shown that the boundaries of the policies move as predicted when the data parameters are changed in a given direction.

\section{Policies structure}

In this section, a parameterized control policy based on the analysis of the numerical results of section 3 is developed. In order to describe the optimal production, replenishment and supplier selection policies by mathematical equations, the boundaries parameters observed and introduced earlier will be used. These parameters are defined as follows:

$Z_{x}^{\alpha}, Z_{y 1}^{\alpha}$ and $Z_{y 2}^{\alpha}$ for the production policy,

$S_{F 1}^{x, \alpha}, S_{F 1}^{y, \alpha}$ and $Q_{F 1}^{\alpha}$ for the replenishment policy from $\mathrm{S} 1$, $S_{F 2}^{x, \alpha}, S_{F 2}^{y, \alpha}$ and $Q_{F 2}^{\alpha}$ for the replenishment policy from $\mathrm{S} 2$.

\subsection{Optimal production policy}

As shown within the numerical results and in Figure 4, the optimal production rate can be described by a Modified Base Stock Policy (MBSP for short) which is state-dependent multilevel, and can be expressed as follows:

$$
p(x, y, \alpha)=\left\{\begin{array}{ccc}
P^{\max } & \text { if } & \left(y<Z_{y 2}^{\alpha} \& x>Z_{x}^{\alpha}\right) \| y<Z_{y 1}^{\alpha} \\
d & \text { if } & Z_{y 1}^{\alpha}<y<Z_{y 2}^{\alpha} \& x<Z_{x}^{\alpha} \\
0 & \text { if } & y>Z_{y 2}^{\alpha}
\end{array}\right.
$$

Recall that $Z_{x}^{\alpha}, Z_{y 1}^{\alpha}$ and $Z_{y 2}^{\alpha}$ represent the threshold parameters with the following constraints.

$$
Z_{x}^{\alpha} \geq 0 ; Z_{y 2}^{\alpha}>Z_{y 1}^{\alpha} \geq 0
$$

\subsection{Optimal replenishment policies}

As shown previously and in Figure 2, the optimal replenishment policies can be described by a State-Dependant Economic Order Quantity policy (SD-EOQ for short) which can be expressed by the following equations.

Replenishment policy from supplier 1:

$$
\Omega^{1}(x, y, \alpha)=\left\{\begin{array}{ccc} 
& & x<S_{F 1}^{x, \alpha} \& \\
Q_{F 1}^{\alpha}(x, y) \quad \text { if } \quad \begin{array}{l} 
\\
\text { otherwise }
\end{array} & \lambda(x, y)=1 \\
0 & &
\end{array}\right.
$$

Replenishment policy from supplier 2:

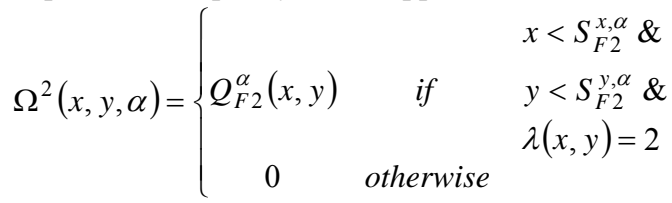

Recall that $S_{F 1}^{x, \alpha}, S_{F 1}^{y, \alpha}$ and $Q_{F 1}^{\alpha}$ (resp., $S_{F 2}^{x, \alpha}, S_{F 2}^{y, \alpha}$ and $Q_{F 2}^{\alpha}$ ) represent the order points and the economic order quantities for supplier 1 (resp. supplier 2), $\lambda$ represent the selection policy indicator, and is defined in section 4.3.

\subsection{Optimal supplier selection policies}

It follows from the replenishment policies observed that the selection policy (see Figure 3) is governed by a StateDependant Up-To-Levels policy, SD-UTL for short, which can be expressed by the following equations.

$\lambda(x, y)=\left\{\begin{array}{ccc}1 & \text { if } & x<S_{F 1}^{X, \alpha} \& y<S_{F 1}^{y, \alpha} \\ 2 & \text { if } \quad S_{F 1}^{x, \alpha}<x<S_{F 2}^{x, \alpha} \& S_{F 1}^{y, \alpha}<y<S_{F 2}^{y, \alpha} \\ 0 & \text { otherwise }\end{array}\right.$

The joint production, replenishment and selection policies, as shown in figure 5, are reactive strategies governed by 9 parameters. These parameters depend on the whole state of the system $(\boldsymbol{x}, \boldsymbol{y}$ and $\boldsymbol{\alpha})$. The order points reflect the necessity to have a security material stock level to face a possible random delivery delay of the selected supplier or a big amount of backlog accumulated after a period of unavailability of the transformation stage. The thresholds governing the production policy reflect also security stock levels to face future periods of unavailability of the transformation stage and the suppliers. 


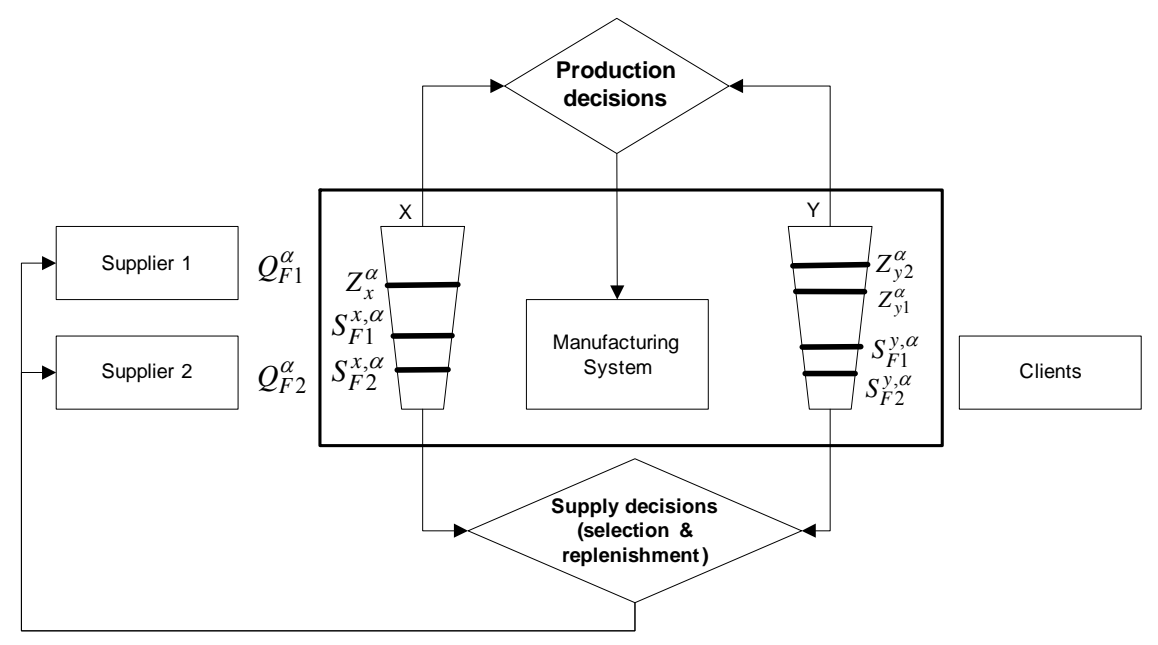

Figure 5. Reactive strategies

\section{COMPARATIVE STUDY AND MANAGERIAL IMPLICATION}

In a highly competitive market, supply chain actors must be involved in a continuous performance improvement process. In this context, the presence of a shared market presses the suppliers to offer the best compromise between price and availability. On the other hand, this reality exhorts the manufacturer manager to continually adjust his replenishment strategy. In addition to a complete strategy of production, replenishment and supplier selection, like the one developed in this paper, two important questions arise: What is the difference in long-term costs between multiple suppliers versus one supplier? If the manager is facing a critical situation requiring that he choose one of two existing suppliers, how does he choose? Is the more available-more expensive supplier better? If so, is that always the case? What will be my decision if the cheapest supplier is improving his availability?

In this section, a comparative study aimed at introducing the aforementioned issues is conducted. For the basic case presented in Tables 1 and 2 (SET III), a sensitivity analysis on the second supplier availability is performed and detailed in Table 3 and Figure 6.

As expected, the results show that it is always more profitable to have more than one supplier even if the two available suppliers provide high availabilities rates. In fact, case 5 for example shows that the incurred cost in the presence of two suppliers is $23 \%$ less than the lowest cost with only one supplier (i.e., 128.83 versus 98.59). Moreover, the sensitivity analysis on the supplier 2 availability rate shows that if the manager is facing the decision to exclude one supplier, he should first measure the impact on the incurred cost. In fact, a switching point between the S1 and S2 curves shows that after a given availability rate (i.e., $\approx 94 \%$, point $\mathrm{A}$ in Figure 6 ) the supplier 2 (i.e., the less expensive one) become the best supplier to choose. In the same vein, and to validate this observation, case 8 show that if the supplier 2 is always available, then there is no need to have a selection strategy (i.e., same costs under S2 or S1 \& S2, point B in Figure 6).

Table 3. Comparative study data : availability variation

\begin{tabular}{|c|c|c|c|c|c|c|}
\hline & $\begin{array}{c}\text { S1 } \\
\text { avail. }\end{array}$ & $\begin{array}{c}\text { S2 } \\
\text { avail. }\end{array}$ & $\begin{array}{c}\text { Manuf. } \\
\text { Avail. }\end{array}$ & $\begin{array}{c}\text { Cost } \\
\text { with } \\
\text { S1 }\end{array}$ & $\begin{array}{c}\text { Cost } \\
\text { with } \\
\text { S2 }\end{array}$ & $\begin{array}{c}\text { Cost } \\
\text { with } \\
\text { S1 \& } \\
\text { S2 }\end{array}$ \\
\hline Base & $97 \%$ & $33 \%$ & $83 \%$ & 128.8 & 232.9 & 106.5 \\
\hline 2 & $97 \%$ & $53 \%$ & $83 \%$ & 128.8 & 201.4 & 104.9 \\
\hline 3 & $97 \%$ & $83 \%$ & $83 \%$ & 128.8 & 149.9 & 102.8 \\
\hline 4 & $97 \%$ & $91 \%$ & $83 \%$ & 128.8 & 135.5 & 100.6 \\
\hline 5 & $97 \%$ & $93 \%$ & $83 \%$ & 128.8 & 130.0 & 98.6 \\
\hline 6 & $97 \%$ & $95 \%$ & $83 \%$ & 128.8 & 123.6 & 98.5 \\
\hline 7 & $97 \%$ & $97 \%$ & $83 \%$ & 128.8 & 112.4 & 98.4 \\
\hline 8 & $97 \%$ & $100 \%$ & $83 \%$ & 128.8 & 97.5 & 97.5 \\
\hline
\end{tabular}

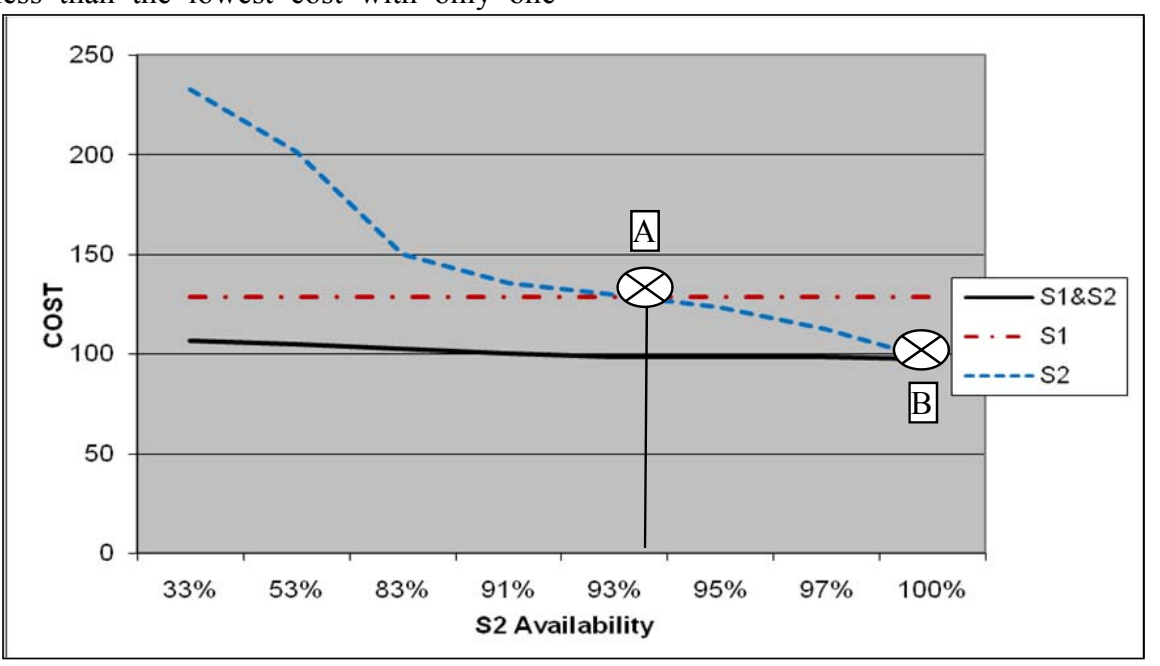

Figure 6: Incurred costs 
To identify the parameters that influence the switching point between the two choices, further sensitivity analyses were conducted. Obviously, if the ordering and raw material costs of supplier 2 decrease, the same sensitivity analysis of Table 3 has shown that the switching point decreases as well. This result follows our expectations since a more available-less expensive supplier is always better. A more interesting result would be to measure the impact of the finished product backlog cost on such decision. Given that the main objective of the supplier chain is to meet demand with the lowest cost, the result presented in table 4 shows that if our customers are less demanding (i.e., lower finished product backlog cost), this gives us an extra margin to choose the least expensive supplier.

Table 4. Impact of $C_{F}^{B}$ on the supplier 2 selection

\begin{tabular}{|c|c|c|c|c|}
\cline { 2 - 5 } \multicolumn{1}{c|}{} & \multicolumn{4}{c|}{ CASES } \\
\cline { 2 - 5 } \multicolumn{1}{c|}{} & BASIC & 2 & 3 & 4 \\
\hline$C_{F}^{B}$ & 5 & 4 & 3 & 2 \\
\hline $\begin{array}{c}\text { S2 AVAIL. RATE } \\
\text { TO SWITCH }\end{array}$ & $94 \%$ & $93 \%$ & $92 \%$ & $91 \%$ \\
\hline
\end{tabular}

In the same direction, in order to measure the impact that the delay duration has on the switching decision and the incurred costs additional sensitivity analysis were conducted. For the fourth case of Table 3 we conducted the same comparative data by varying the replenishment lead time of supplier 2 . Table 5 and Figure 7 show the results obtained.

Table 5. Comparative study data : lead time variation

\begin{tabular}{|c|c|c|c|c|c|}
\hline & $\delta^{1}$ & $\delta^{2}$ & $\begin{array}{c}\text { Cost } \\
\text { with S1 }\end{array}$ & $\begin{array}{c}\text { Cost } \\
\text { with S2 }\end{array}$ & $\begin{array}{c}\text { Cost } \\
\text { with S1 } \\
\& \text { S2 }\end{array}$ \\
\hline Basic & 0.1 & 0.4 & 128.83 & 135.56 & 100.60 \\
\hline 2 & 0.1 & 0.3 & 128.83 & 133,58 & 100,11 \\
\hline 3 & 0.1 & 0.2 & 128.83 & 131,62 & 99,62 \\
\hline 4 & 0.1 & 0.1 & 128.83 & 129,69 & 99,13 \\
\hline 5 & 0.1 & 0.05 & 128.83 & 127,79 & 98,64 \\
\hline 6 & 0.1 & 0.03 & 128.83 & 125,92 & 98,16 \\
\hline
\end{tabular}

As expected, the results show that it is always more profitable to have more than one supplier. However, when the system has to run with only one supplier it clearly appears that the lead time of the suppliers has a significant effect on the decision to choose one of the two available suppliers. In fact, a switching point between the $\mathrm{S} 1$ and $\mathrm{S} 2$ curves shows that when the supplier 2 lead time decreases and after a given value (i.e., $\approx 0.07$, point $\mathrm{A}$ in Figure 7 ) the supplier 2 (i.e., the less expensive one) become the best supplier to choose.

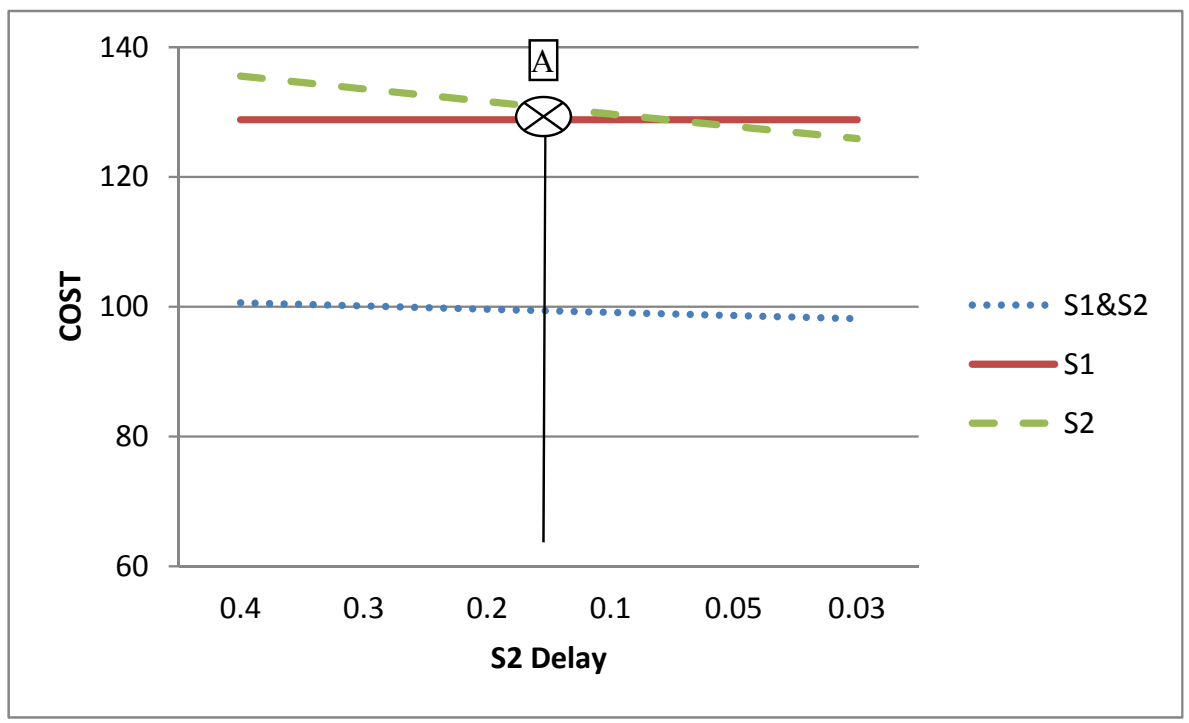

Figure 7: Incurred costs: lead time variation

\section{Conclusion}

In conclusion, this paper makes an important contribution to the discourse as it develops complete information sharing production, replenishment and supplier selection control policies for an unreliable supply chain. These policies are described by equation (8) to (12), and illustrated in Figure 2, 3 and 4 . The policies are "information sharing" since the supply chain actors establish their control strategies over the entire state space.

From a mathematical perspective, we invoked optimal and impulsive control theory notions to propose a hybrid stochastic model of the problem considered. We have numerically solved the Hamilton Jacobi Bellman equations of the problem and carried out deep sensitivity analysis. Based on the numerical results obtained, a sub-optimal control policy for the problem was derived, and is shown to be described by a combination MBSP and SD-EOQ, SD-UTL policies defined by nine parameters for the case of two suppliers and $3 \times(n+1)$ for the case of $n$ suppliers.

A thorough sensitivity analysis, conducted on the main parameters characterizing a given supplier (i.e., availability rates, lead time and costs), shows that it is always better to operate with multiple suppliers and to base production and 
replenishment activities on the control policies developed. Such a strategy guarantees costs that are up to $28 \%$ lower. Furthermore, the presence of a switching point between the choice of one supplier over another reveals the need in such situation to consider the dynamic stochastic aspects of the whole system in an integrated manner so as to make the best decisions.

\section{REFERENCES}

Capuzzo-Dolcetta, I. and Lions, P.L., 1990. Hamilton-Jacobi equations with state constraints. Transactions of the American Mathematical Society, 318 (2), 643-683.

Chan, H.K., and Chan, F.T.S., 2009, "A review of coordination studies in the context of supply chain dynamics", International Journal of Production Research, under press.

Choi, T.Y., and Hartley, J.L., 1996, "An exploration of supplier selection practices across the supply chain," Journal of Operations management, vol. 14, pp. 333-343.

Chuang, C.L., 2004, « Supplier selection and order allocation in supply chain management », Ph. D. Thesis, the university of Iowa.

Fong, D.K.H., Gempesaw, V.M. and Ord, J.K., 2000, "Analysis of a dual sourcing inventory model with normal unit demand and Erlang mixture lead times" European Journal of Operational Research, 120, pp. 97-107.

Ghodsypour, S.H., and O'Brien, C., 1998, “A decision support system for supplier selection using an integrated analytic hierarchy process and linear programming," Int. J. Prod. Econ., vol. 56-57, pp. 199-212.

Hajji A, Gharbi A. and Kenne J. P, 2009, « Joint replenishment and manufacturing activities control in two stages unreliable supply chain », International Journal of Production Research, 47(12), 3231-3251.

Johansen, S.G., and Thorstenson, A., 1998, "An inventory model with Poisson demands and emergency orders" International Journal of Production Economics, 56-57, pp. 275-289.

Lee, E.K., Ha, S., and Kim, S.K., 2001, "Supplier selection and management system considering relationships in supply chain management," IEEE Transactions on Engineering Management, vol. 48, no. 3, pp. 307-318.

Minner, S., 2003, "Multiple-supplier inventory models in supply chain management: A review," Int. J. Production Economics, 81-82, pp. 265-279.

Moinzadeh, K., and Nahmias, S., 1988, "A continuous review model for an inventory system with two supply modes" Management Science, 34, pp. 761-773.

Parlar, M., and Perry, D., 1995, "Analysis of a (Q, r, T) inventory policy with deterministic and random yields when future supply is uncertain". European Journal of Operational Research, 84, pp. 431-443.

Parlar, M., and Perry, D., 1996, "Inventory models of future supply uncertainty with single and multiple suppliers". Naval Research Logistics, 43, pp. 191-210.

Syam, S. and Shetty, B., 1998, "Coordinated replenishments from multiple suppliers with price discounts". Naval Research Logistics, 45, pp. 579-598.

Tan, K.C., Kannan, V.R., and Handfield, R.B., 1998, "Supply chain management: Supplier performance and firm performance," Int. J. Purchas. Mater. Manage., vol. 34, no. 3, pp. 2-9.

Verma, R., and Pullman, M.E., 1998, "An analysis of the supplier selection process" Omega International Journal of Management Science, vol. 26, no. 6, pp. 739-750.
Zhang, V.L., 1996, "Ordering policies for an inventory system with three supply modes" Naval Research Logistics, 43, pp. 691-708.. 